\title{
Correlation of Sex and Remission of Acute Lymphoblastic Leukemia-L1 (ALL-L1) in Children
}

\author{
Trisna Silawati*, Nadirah Rasyid Ridha, Dasril Daud \\ Department of Pediatrics, Medical Faculty, Hasanuddin University, Makassar, South Sulawesi, Indonesia \\ Email address: \\ Trisnasilawati@gmail.com (T. Silawati),nadirah.bika@gmail.com (N. R. Ridha),drdasril@gmail.com (D. Daud)
}

\section{To cite this article:}

Trisna Silawati, Nadirah Rasyid Ridha, Dasril Daud. Correlation of Sex and Remission of Acute Lymphoblastic Leukemia-L1 (ALL-L1) in Children. International Journal of Clinical and Experimental Medical Sciences. Vol. 1, No. 2, 2015, pp. 11-15.

doi: 10.11648/j.ijcems.20150102.12

\begin{abstract}
Introduction: Acute Lymphoblastic Leukemia (ALL) is a malignancy often found in children with peak incidence of 2-5 years. The symptoms and biological factors affect the ALL prognosis; age, leukocyte count, sex, nutritional state, ALL phenotype, CNS infiltration, chromosome abnormalities, and early response to chemotherapy. Objective: The aim of this study was to determine the role of sex on the patient's remission with ALL-L1. Methods: The retrospective cohort study was conducted to determine the role of sex on the patient's remission with ALL-L1. The study was conducted in the pediatric ward of Dr. Wahidin Sudirohusodo hospital in Makassar from March until November 2014. The cases were taken from the pediatric ward that met both the inclusion and exclusion criteria. The data were taken from the patient's medical records related to the study. Results: Revealed of 70 cases that met the criteria was consisted of 42 males and 28 females. Frequency of remission in male group was $41(97.6 \%)$ and in female group was $27(96.4 \%)$. The statistical analysis revealed that there was no significant difference between sex and remission, as it was shown by $p=0.643(p>0.05)$. Conclusion: Sex did not have any effects on the frequency of remission in children with ALL-L1 in Dr. Wahidin Sudirohusodo hospital. The frequency of remission in male group was $97.6 \%$, and in female group was $96.4 \%$.
\end{abstract}

Keywords: Sex, Remission, ALL-L1, Child

\section{Introduction}

Until nowadays, many studies have not been able to explain the influence of sex on remission of patients with ALL- L1 (acute lymphoblastic leukemia-L1). ALL-L1 is a clonal malignancy of lymphoid precursor cells. Both male and female can experience the chromosome translocations in leukemia resulting excessive deregulation of gene expression or the formation of a new gene fusion, aneuploidy and specific gene mutations. There are several factors can influence the prognosis of patients that can explain why the ALL-L1 in males is worse than females. They are such as high leukocyte count, older age, T cells immunophenotype, infiltration of the central nervous system, and the Philadelphia translocation. ${ }^{1}$ Another factor is the difference in the metabolism of lymphoblast in the maintenance phase of chemotherapy regimens between males and females. The difference is due to differences in metabolic enzyme activity in derived sex chain. ${ }^{2,}{ }^{3}$ In addition, the blood testicular barrier associated with relapse because the blood testicular barrier can protect lymphoblast from exposure to chemotherapy. Testicular relapse is often followed by a bone marrow relapse. ${ }^{2,4}$ Therefore, it is important to investigate the influence of sex on remission of the ALL-L1 patient.

However, some research suggest various facts that sex is a significant prognostic factor in ALL-L1. According to Nimeyer et al. Males have worse outcomes than females that get the same leukemia therapy. ${ }^{5}$ Meanwhile, Keizo Horibe et al. found that the Philadelphia chromosome, a translocation of chromosomes, leukocytes $>100.000 / \mathrm{L}$ and male sex prognosis is less satisfactory. ${ }^{6}$ Meanwhile, in the Department of Child Health, University of Indonesia in 2009 found that there was no difference in survival rates between males and females also similar result was obtained by the Department of Pediatrics, Hasanuddin University in Makassar in 2012. ${ }^{7,8}$ Sex is no longer considered as a significant prognostic factor in patients with newly leukemia diagnosis. ${ }^{9}$ According to Pui $\mathrm{CH}$, although males and females got benefit by an improvement in the ALL-L1 therapy it cannot be denied that the females had 10-year EFS better than males with $73.4 \pm$ $3.7 \%$ v $63.5 \% \pm 4 \%{ }^{2}$

In Indonesia, especially in South Sulawesi the study of sex 
and its relation with remission of the ALL-L1 in children has never been done yet. The purpose of this study was to determine whether sex has an influence on remission of ALL-L1 patients or not.

\section{Methods}

This was a retrospective cohort study to assess the relationship of sex with ALL-L1 remission in children. This study was conducted at Dr. Wahidin Sudirohusodo Hospital in Makassar from March to November 2014, using the data from medical records of patients with ALL-L1 that were registered in hemato-oncology division of Pediatrics Department of Dr. Wahidin Sudirohusodo that uses the 2006 ALL protocol. All the eligible population were the ALL-L1 patients in the induction phase after obtaining the induction chemotherapy with the same protocol. This study has been approved by the Ethics and Research Committee of the Hospital and Hasanuddin University. Written informed consent was obtained from the patients' parents or legal guardian following full and detailed explanation regarding the study protocol.

We consecutively chose the patients from all the eligible population who met the inclusion and exclusion criteria. Determination of case size was based on the desired confidence level that was $95 \%$. By this we mean that when the proportion of patients with leukemia in the population is $20 \%$, the desired level of absolute immutability (d) is $10 \%$ with a significant level $(\alpha)$ of 1.96 and $Q=(1-P)$, then the estimated case size in this study was determined minimum of 63 people. Based on patient's medical record, we recorded all the data that had a correlation to our study. The inclusion criteria were all the ALL-L1 patients that were registered in hemato-oncology division of Pediatrics Department in the induction phase whom were treated by chemotherapy with the same protocol and $>1$ year of age. While, the exclusion criteria were the patients with other types of ALL. Then, we divided the patients into two groups; the group of ALL-L1 of males and females. We observed both of the groups' outcomes that had remission or not. The remission in our study was not the discovery of leukemia blast cells in the blood or cerebrospinal fluid, and the result of bone marrow plasma (BMP) lymphoblast was $\leq 5 \%$ and there was no evidence of extramedullary diseases or symptoms; after performing the induction phase a bone marrow aspiration control was recorded in the medical record. The entire patients in both groups were recorded their registered number, sex, aged on diagnosis, body mass index, mediastinal mass, hyperleukocytosis, the infiltration to central nervous system and bone marrow, the complete or not remission after the induction. The data were obtained from the medical records were grouped by purpose and type. The data were analyzed with appropriate statistical methods, such as univariate and bivariate analysis consisting of student $t$ test and $\mathrm{X}^{2}$ test (Chisquare) or Fisher's exact test with the SPSS version 17.

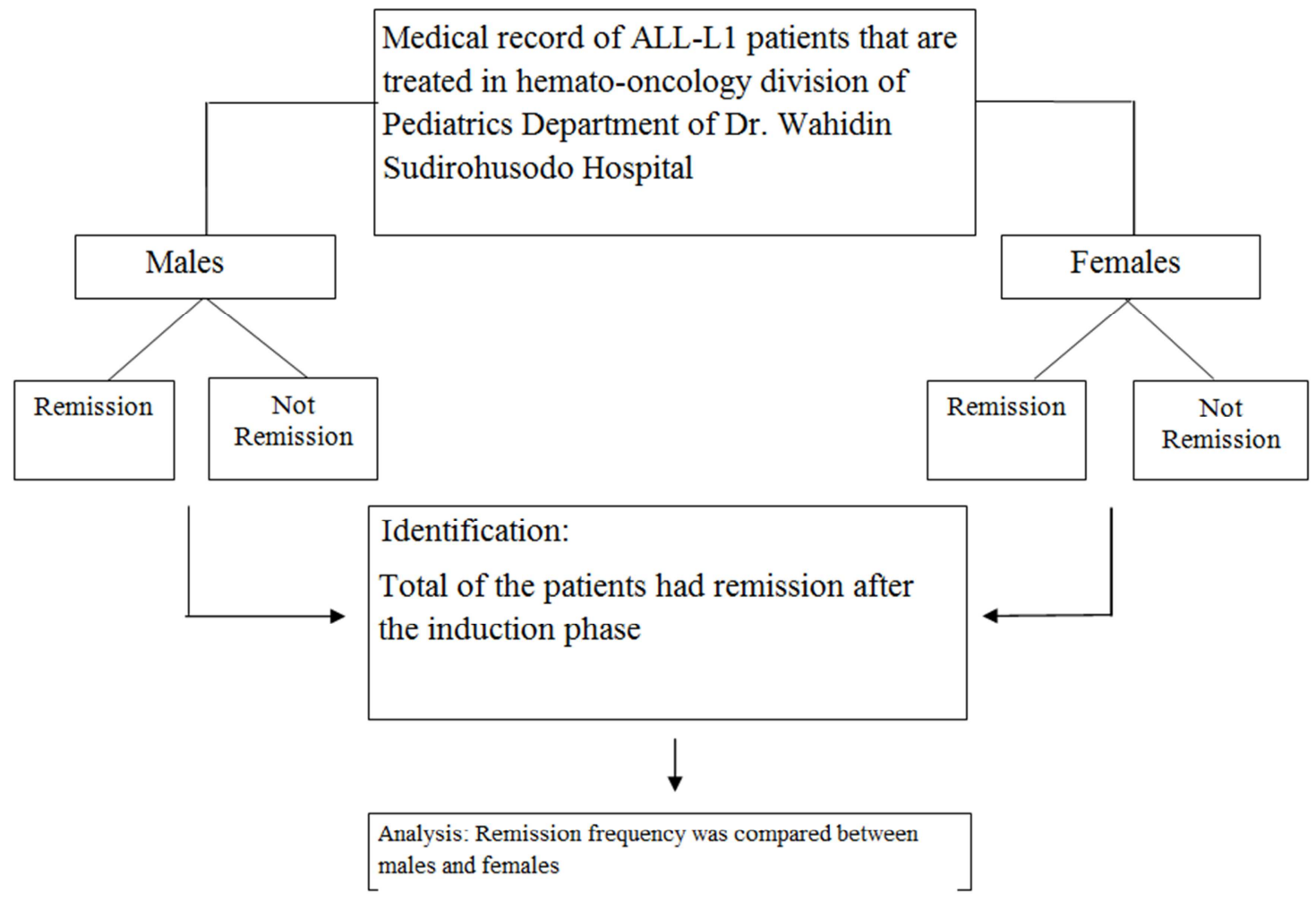

Figure 1. Study flow scheme. 


\section{Results}

The results showed, of the 70 cases that met the criteria 42 patients were male and 28 patients were female. The remission frequency in the male group was 41 patients $(97.6 \%)$ and in the female group was 27 patients $(96.4 \%)$ with p-value $>0.05$.

Table 1. Case characteristics.

\begin{tabular}{|c|c|c|}
\hline \multirow{3}{*}{1.} & $\operatorname{Sex}(\%)$ & \\
\hline & Male & $42(60.0)$ \\
\hline & Female & $28(40.0)$ \\
\hline \multirow{3}{*}{2.} & Remission (\%) & \\
\hline & Remission & $68(97.1)$ \\
\hline & Not remission & $2(2.9)$ \\
\hline \multirow{3}{*}{3.} & Protocol & \\
\hline & Standard risk & 45 (64.3) \\
\hline & High risk & $25(35.7)$ \\
\hline
\end{tabular}

Table 2. The relationship of sex with remission.

\begin{tabular}{llll}
\hline \multirow{2}{*}{ Sex } & \multicolumn{2}{c}{ Total } \\
\cline { 2 - 3 } & Remission & Not remission & \\
\hline Male & $41(97.6 \%)$ & $1(2.4 \%)$ & $42(100 \%)$ \\
Female & $27(96.4 \%)$ & $1(3.6 \%)$ & $28(100 \%)$ \\
Total & $68(97.1 \%)$ & $2(2.9 \%)$ & $70(100 \%)$ \\
\hline
\end{tabular}

Fischer's Exact Test df $=1 \mathrm{p}=0.643(\mathrm{p}>0.05)$

Statistical analysis in table 2 shows the remission frequency of the male group was 41 patients $(97.6 \%)$ and the female group was 27 patients $(96.4 \%)$ and the not remission frequency in the male group was 1 patient $(2.4 \%)$ and the female group was also 1 patient $(3.6 \%)$. There was no difference of the remission frequency between the male and female group, with $p=0.643(p>0.05)$. It means that the ALL patients' frequency with remission and not remission in both groups had no meaningful difference.

Table 3. The relationship of sex with the treatment protocol.

\begin{tabular}{llll}
\hline \multirow{2}{*}{ Sex } & \multicolumn{3}{l}{ Total } \\
\cline { 2 - 3 } & High Risk & Standard Risk & \\
\hline Male & $18(42.9 \%)$ & $24(57.1 \%)$ & $42(100 \%)$ \\
Female & $7(25.0 \%)$ & $21(75.0 \%)$ & $28(100 \%)$ \\
Total & $25(64.3 \%)$ & $45(35.7 \%)$ & $70(100 \%)$ \\
\hline
\end{tabular}

Chi Square Test $\mathrm{df}=1 \mathrm{p}=0.127(\mathrm{p}>0.05)$

Statistical analysis in table 3 shows the patients' frequency with the high risk of treatment protocol in the male group was 18 patients $(42.9 \%)$ and in the female group was 7 patients $(25.0 \%)$. While the patients' frequency with the standard risk in the male group was 24 patients $(57.1 \%)$ and in the female group was 21 patients $(75.0 \%)$. The statistical analysis showed there was no meaningful difference between sex and treatment protocol, with $\mathrm{p}=0.127(\mathrm{p}>0.05)$. It means that the ALL patients' frequency with the high risk and standard risk treatment protocol in both groups had no meaningful difference.
Table 4. The relationship of treatment protocol and remission with the male sex

\begin{tabular}{llll}
\hline \multirow{2}{*}{ Protocol } & \multicolumn{2}{c}{ Total } \\
\cline { 2 - 3 } & Remission & Not remission & \\
\hline Standard Risk & $24(100 \%)$ & $0(0 \%)$ & $24(100 \%)$ \\
High Risk & $17(94.4 \%)$ & $1(5.6 \%)$ & $18(100 \%)$ \\
Total & $41(97.6 \%)$ & $1(2.4 \%)$ & $42(100 \%)$ \\
\hline
\end{tabular}

Fischer's Exact Test df $=1 \mathrm{p}=0.440(\mathrm{p}>0.05)$

Statistical analysis in table 4 shows that the ALL male patients' frequency with standard risk treatment protocol was 24 patients $(57.1 \%)$ and with high risk treatment protocol was 18 patients $(42.9 \%)$. The patients' frequency with standard risk treatment protocol in the remission group was 24 patients $(100 \%)$ and in not remission group was zero $(0 \%)$. The frequency of patients with high risk treatment in the remission group was 17 patients (94.4\%) and in not remission group was 1 patient $(5.6 \%)$. The statistical analysis showed there was no meaningful difference between the treatment protocols and remission occurrence, with $\mathrm{p}$-value $=$ $0.440(p>0.05)$. It means that the frequency of the ALL male patients who got the high risk and standard risk treatment protocol in both groups with remission and not remission had no meaningful difference.

Table 5. The relationship of treatment protocol and remission with the female sex.

\begin{tabular}{llll}
\hline \multirow{2}{*}{ Protocol } & \multicolumn{2}{c}{ Total } \\
\cline { 2 - 3 } & Remission & Not remission & \\
\hline Standard Risk & $20(95.2 \%)$ & $1(4.8 \%)$ & $21(100 \%)$ \\
High Risk & $7(100 \%)$ & $0(0 \%)$ & $7(100 \%)$ \\
Total & $27(96.4 \%)$ & $1(3.6 \%)$ & $28(100 \%)$ \\
\hline
\end{tabular}

Fischer's Exact Test df $=1 \mathrm{p}=0.429(\mathrm{p}>0.05)$

Statistical analysis in table 5 shows that the ALL female patients' frequency with standard risk treatment protocol was 21 patients $(75 \%)$ and with high risk treatment protocol was 7 patients $(25 \%)$. The frequency of patients with standard risk treatment in remission group was 20 patients $(95.2 \%)$, while in not remission group was 1 patient $(4.8 \%)$. The patients' frequency of high risk treatment protocol with remission was 7 patients $(100 \%)$ and in not remission group was zero $(0 \%)$. The analysis showed there was no meaningful difference between the treatment protocols and remission occurrence, with $p$-value $=0.429(p>0.05)$. It means that the frequency of the ALL female patients who got the high risk and standard risk treatment protocol in both groups with remission and not remission had no meaningful difference.

\section{Discussion}

Remission is the main goal in the ALL treatment. Since the last thirty years, ALL treatment in children has been a significant improvement signed by the reach of event-free survival (EFS) of $80 \%$ and overall survival time (OST) of $90 \%$. This is the result of patient management improvement by using a multidrug chemotherapy and treatment implementation according to the risk stratification of patient 
group. The latest treatment strategy according to the genetic characteristic and molecular of leukemia such as immunophenotype, chromosome abnormality, and detection of residual disease till several of the conventional prognostic factors like sex is not significant as an ALL outer predictor factor anymore.

This study used a retrospective cohort design with data from the medical records to assess the sex role on the remission of ALL patients. There were 70 cases, which were 42 males and 28 females. Then, they were analyzed to assess the patient remission difference that was based on the sex. In the bivariate analysis of factors that were related to the sex, there was no meaningful relationship between the sex with the early leukocyte counts, ALL risk group, remission or not remission at the end of induction phase with p-value $>0.05$.

The difference in survival of children with ALL based on the sex has been reported in several literatures. Male children showed the less survival than the female ones. In this study, the male remission was $97.6 \%$ and the female remission was $96.4 \%$, with $\mathrm{p}$-value $=0.643(\mathrm{p}>0.05)$. In 2009, Rana et al. obtained from 38 patients that there were 28 patients $(74 \%)$ that experienced the complete remission, 2 patients $(5 \%)$ had partial remission, and 1 patient $(3 \%)$ had not remission. ${ }^{10}$

The role factor in minimizing the ALL patients remission difference is the ALL treatment protocols based on the ALL prognostic factor stratification not only based on clinical, but also based on the immunophenotype examination, cytogenetic and molecular. In 1994, ALL patients in the high risk group had hyperleukocytosis, leukemia central nervous system, mediastinal masses, ALL-L3 and ALL relapse. In 2006, ALL high risk prognostic factors were aged $<1$ year or $>10$ years, ALL relapse, hyperleukocytosis, mediastinal mass, leukemia central nervous system, T cell leukemia and mixed leukemia. From the two ALL treatment protocols in Indonesia, there was no sex include in the ALL prognostic. This means that the ALL treatment stratification is not based on the sex, so from the study obtained there was no remission difference in ALL patients between male and female children. The whole study means that the sex has no effects on the remission in the ALL patients.

In this study, the ALL patients remission was higher than the study result in the developing country because of the successful chemotherapy by using the ALL chemotherapy protocol in 2006 with multidrug chemotherapy, in addition of good supportive treatment. In 2009, obtained from 38 patients, 7 patients $(18 \%)$ died because of neutropenia and sepsis along the induction phase chemotherapy. ${ }^{10}$ Most of the patients died in the induction phase because of infection or hemorrhage. The role factor in this incident was the lack of facilities for supportive treatment of ALL patients, such as the special isolation room for the infected patients because of neutropenia. Nowadays, with the better procedure and treatment in the Dr. Wahidin Sudirohusodo, and also the social security agency insurance, we hope the outer ALL patients' data improve.

Factor affects the difference in the ALL patients between the male and female children becomes not meaningful is a reduction of the relapse. The intensive ALL therapy with a high dose of MTX (methotrexate) can reduce the testicular relapse on ALL. Niemeyer et al. said with the high dose of MTX, the testicular relapse incidence decreased from $20 \%$ to $4 \%$. The another relapse source is leukemia central nervous system. ${ }^{5}$ With the use of intrathecal regimens directly in the central nervous system, namely MTX and cytarabine chemotherapy, the incidence of central nervous system relapse reduced.

In this study, the immunophenotype and cytogenetic examine on the ALL patients had not become the routine treatment procedure yet, because of the high charge. Thus, the immunophenotype and cytogenetic probably affect the sex could not be analyzed. It maybe has a role to make no difference to the ALL patients in this study.

The strength of the study was using a secondary data with many cases by the retrospective cohort study method as the first in South Sulawesi to assess the sex influence on the ALL patients remission, thus can be considered in classification of ALL patient risk group. The other strength of this study was the hospital (Dr. Wahidin Sudirohusodo) is the biggest hospital in the East Indonesia. This means that the ALL patients in the hospital came from various places in East Indonesia. Therefore, this study result can be a reference or generalization to get the description of the ALL patients' survival in Indonesia.

The constraint in this study was using data from the medical record so were found some shortcomings, which were the incomplete data. This was one of the causes that many cases were missed from the observations (loss of follow-up); therefore, the unknown status of their life as many as 35 cases.

According to the study, we conclude that the sex does not affect the ALL-L1 remission in children, which is the male remission number was $97.6 \%$ and the female remission was $96.4 \%$. Based on the study result, we recommend the study result as a basic that the chemotherapy treatment is not stratified according to the sex and doing a study with larger number of cases. As well as this, medical records of the ALL patients need to be made more complete to facilitate the tracking patient record for research purposes.

\section{References}

[1] Schuster JJ., Walker P., Pullen J., Humbert J., Land VJ., Mahoney DH., et al. Prognostic significance of sex in childhood $\mathrm{B}=$ Precursor acute lymphoblastic leukemia: A Pediatric Oncology Group Study. J Clin oncol. 1998; 16: 2854-2863.

[2] Pui CH., Boyett JM., Relling MV. Sex differences in prognosis for children with acute lymphoblastic leukemia. Journal of Clinical Oncology. 1999; 17 (3): 818-24.

[3] Chessells JM., Hall E., Pretinence HGM., Bailey CC., Richards SM. The impact of age on outcome in lymphoblastic leukemia; MRC UKALL X and XA compared: a report from the MRC Pediatric and Adult Working Parties. Leukemia. 1998; 12: 463-73. 
[4] Margolin JR, Stember CP, Poplack DG. Acute lymphoblastic leukemia, In: Pizzo PA, Poplack DG, Eds. Principles and practice of pediatric Oncology. Philadelpia. 2002; 489-544.

[5] Niemeyer CM., Reiter A., Riehm H. Comparative results of two intensive treatment programs for childhood acute lymphoblastic leukemia: The Berlin-Frankfurt-Munster and Dana-Farber Cancer Institute Protocols. Ann Oncol. 1991; 2: 745-749.

[6] Reiter A., Schrappe M., Ludwig WD., et al. Chemotherapy in 998 unselected childhood acute lymphoblastic leukemia patients: results and conclusion of the multicenter trial ALLBFM 86. Blood. 1994; 84:3122-33.

[7] Permatasari E., Widyastuti E., Satari H.I. Survival and prognostic factors of childhood acute lymphoblastic leukemia. Paediatrica Indonesiana. 2009; 49 (6): 365-71.

[8] Haryani, Yeni. Sex and survival variability in children with acute lymphoblastic leukemia. PPDS of Medical Faculty, Hasanuddin University. 2013; 51-57.

[9] Coustan-Smith E., Behm F.G., Sanchez J., Boyett J.M. Immunological detection of minimal residual disease in children with acute lymphoblastic leukemia. Lancet. 1998; 21: 550-04.

[10] Rana Ali. Outcome of childhood acute lymphoblastic leukemia after induction therapy-3 years experience at a single paediatric oncology centre. J Ayub Med Coll Abbottabad. 2009; 21(4):150-3. 